\title{
75. Discrimination of Amino Acid by Anticodon and Discriminator Base
}

\author{
By Mikio Shimizu \\ Institute of Space and Aeronautical Science, University of Tokyo, \\ Komaba, Meguro-ku, Tokyo 153
}

(Communicated by Masao KotANI, M. J. A., Oct. 12, 1979)

The central dogma of molecular biology suggests the sequence of DNA-mRNA-tRNA-protein amino acid to determine the heredityenzyme system relation of the living system. In the sequence, the mode is unknown as to how the anticodon on tRNA discriminates the amino acid, whose position is far from that of the anticodon. The 'discriminator base' is a nucleic acid base at the 4th position of 3 ' end of tRNA and recognizes a specific group of protein amino acids. ${ }^{1), 2)}$ This base is also far from the anticodon and their mutual relation has not yet been understood.

I have examined whether or not a complex consisting of an anticodon and the corresponding discriminator base (in the case of $E$. Coli) can discriminate the corresponding amino acid. In this complex of four nucleotides $(\mathrm{C} 4 \mathrm{~N})$, the nucleic acid bases in the anticodon are assumed to be in anti form and to use their three hydrogen bonds (one bond from each base) to combine with three sites on the discriminator base. When the discriminator base is guanine or cytosine, the three sites are the same sites as to form three hydrogen bonds in DNA. In the case of uracil, they are at the positions of 2,3 , and 4 and in the case of adenine, they are at the positions of 1,3 , and 6 (Figs. 1-8). I found that the holes formed by the C4N or those formed by the third and second nucleotides in the anticodon (corresponding to the first and second nucleotides in the codon) with the discriminator base were very well matched to the side chains of the protein amino acids in the relation of key-key hole. It is interesting that, in this structure, most of hydrogen atoms in the side chain of the amino acid are in the vicinity of oxygen and nitrogen atoms in the $\mathrm{C} 4 \mathrm{~N}$ for stabilizing the whole system. I have ascertained these relations for all twenty protein amino acids by using the CoreyPauling-Koltun molecular model.

For instance, the side chain of valine $\left(-\mathrm{CH}\left(\mathrm{CH}_{3}\right)_{2}\right)$ or tyrosine $\left(-\mathrm{CH}_{2}-\square-\mathrm{OH}\right)$ fits exactly into the holes of the complex $\mathrm{CA}=\mathrm{A}$ or $\mathrm{AUA} \equiv \mathrm{A}$, respectively. In the case of the code which terminates the peptide chain with a codon UAA or UAG whose discriminator base 


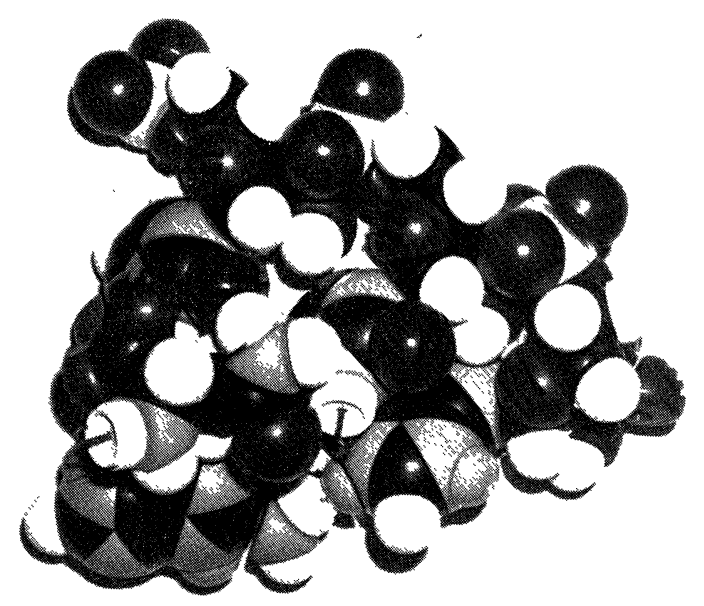

Fig. 1. $\mathrm{C} 4 \mathrm{~N}$ of $\mathrm{UUA} \equiv \mathrm{G}$ with asparagine on it. The discriminator base is shown only in the form of nucleic acid base. The CCA bond which starts from the nucleotide bond of the discriminator base is so flexible that it can easily reach the peptide bond of the amino acid at any configuration on the C4N. The acceptor stem of tRNA is located in the lower right corner of the figure without disturbing the above configuration.

could be taken to be $A$ as in the case of tyrosine with a codon UAU or UAC, the hole is too small to fit any of nineteen side chains and also too large to fit that of glysine.

In the above $\mathrm{C} 4 \mathrm{~N}$ model, the genetic code should be written in terms of three (not two or five) nucleic acid bases, since the discriminator base has three sites on it to connect with the anticodon through hydrogen bonds.

There remain many problems to be studied on the origin and the evolution of the genetic code. For instance, we should find the mode how to combine the anticodon and the discriminator which are far from each other on the present tRNA. However, Hopfield ${ }^{3)}$ discussed recently that the anticodon of a primitive tRNA in the early history of the earth would have been in the vicinity of the discriminator base and have directly formed a cage of the amino acid by analysis of the structure of the present tRNA, although he did not take into account the role of the discriminator base. H. Mizuno suggested me, in the symposium on the origin of protein synthesis system at Kumatori on August 23-24, 1979, a possibility that a pair of tRNA reversely combined with each other to form the $\mathrm{C} 4 \mathrm{~N}$ at one end might be used by 

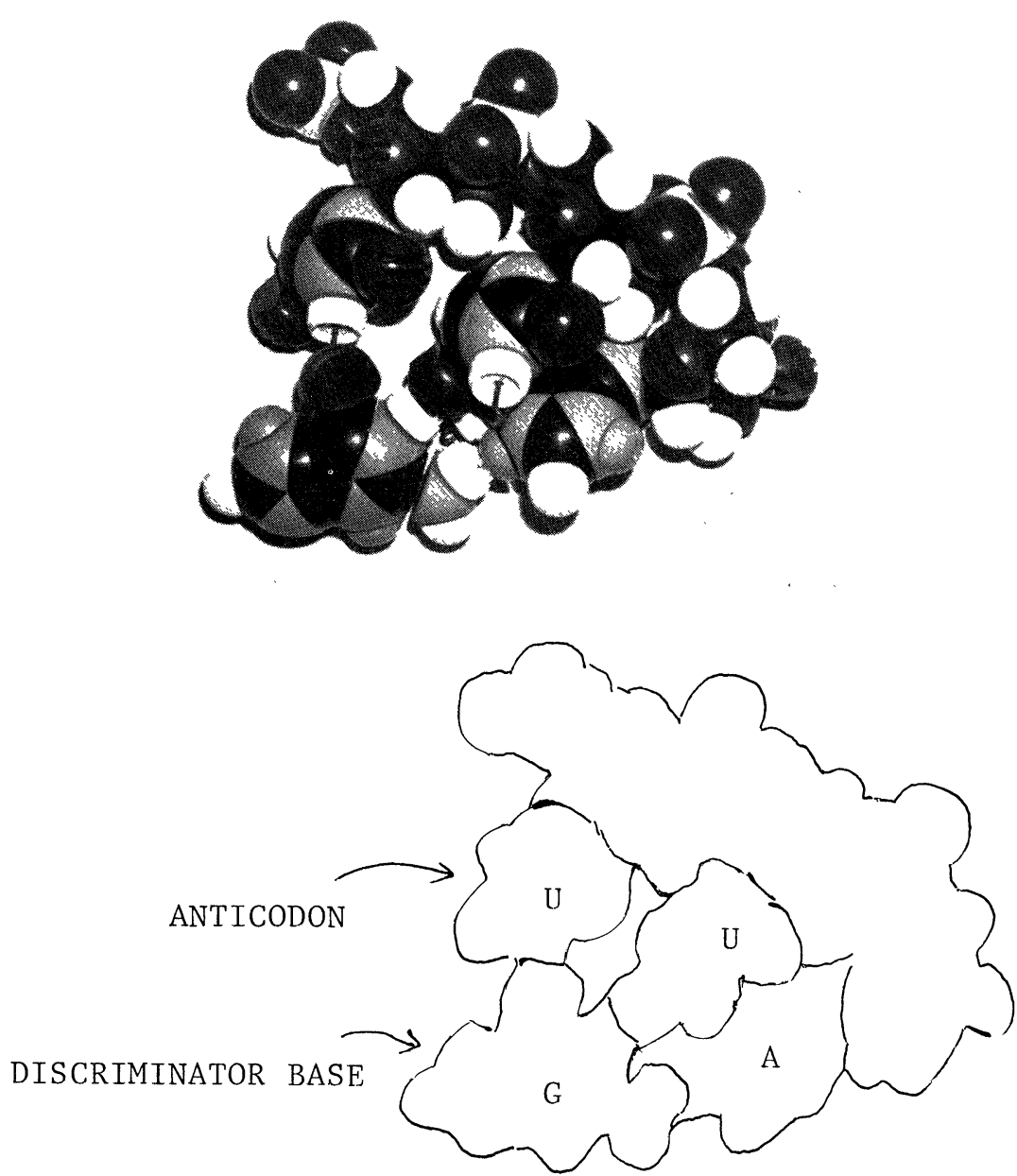

Fig. 2. $\mathrm{C} 4 \mathrm{~N}$ of $\mathrm{UUA} \equiv \mathrm{G}$ without asparagine. The positions of anticodon and discriminator base are shown below.

the aminoacil synthetase to discriminate the corresponding amino acid. Then the discriminator-anticodon complex might have a biological meaning even at present. Consequently I believe that the physical correspondence between the $\mathrm{C} 4 \mathrm{~N}$ and the protein amino acid pointed above will play a crucial role to the understanding of the origin and evolution of the genetic code.

An indirect experimental evidence for the $\mathrm{C} 4 \mathrm{~N}$ formation and the trap of the amino acid by this complex is found in the study of Hayashi and Miura ${ }^{4)}$ on an inhibitory effect of oligonucleotides for tRNA synthetase. The rate of the formation of phenylalanyl tRNA was found to be very strongly inhibited by the addition of oligo-A and 


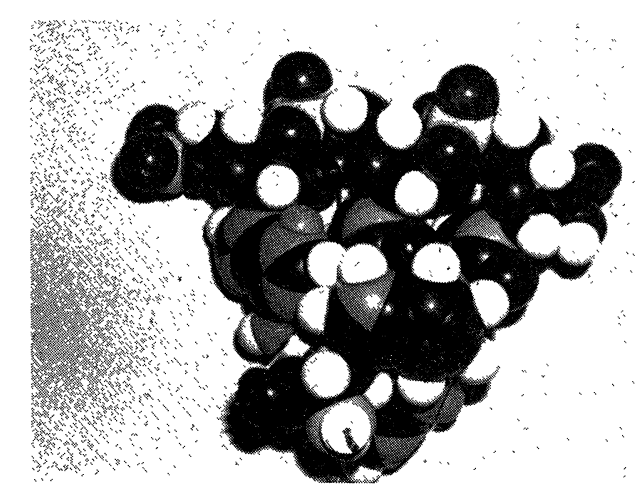

Fig. 3. $\mathrm{C} 4 \mathrm{~N}$ of $\mathrm{ACC} \equiv \mathrm{G}$ with tryptophane on it.

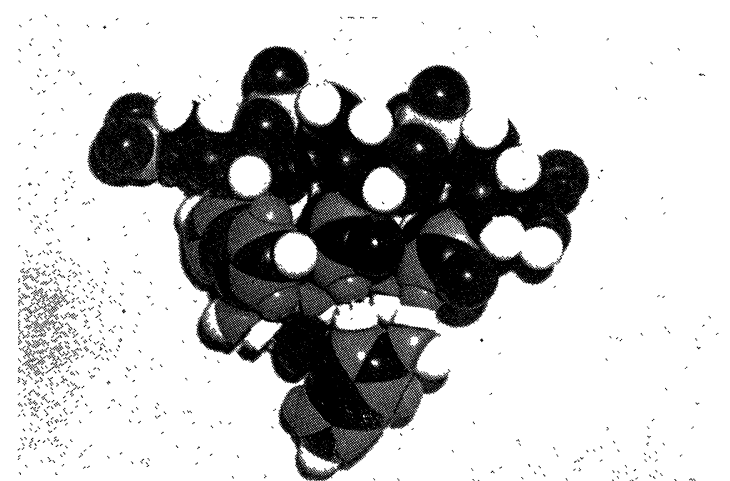

Fig. 4. $\mathrm{C} 4 \mathrm{~N}$ of $\mathrm{ACC} \equiv \mathrm{G}$ without tryptophane.

there was little effect for oligo-U and oligo-C. The rate of lysine incorporation into tRNA was considerably inhibited by oligo-U and there was little effect for oligo-A and oligo-C. The length of the oligonucleotides were 5 or 6 in average in this experiment. In this case, an A triplet at one end of the oligo-A may be expected to form a $\mathrm{C} 4 \mathrm{~N}$, $(\mathrm{AAA} \equiv \mathrm{A})$, with an $\mathrm{A}$ at the other end of the oligo-A. Consequently, the result of the experiment on phenylalanine could be interpreted in terms of the formation of the (AAA $\equiv \mathrm{A})$ and phenylalanine system. The amino acid was absorbed by the $\mathrm{C} 4 \mathrm{~N}$ and tRNA could not form aminoacil tRNA even in the presence of synthetase. Similarly the weak inhibitory effect of oligo-U on lysine may be explained by the formation of a $\mathrm{C} 4 \mathrm{~N}$ (UUU $\equiv \mathrm{U}$ ), in which the discriminator base is not correctly $\mathrm{A}$ but $\mathrm{U}$, and lysine incomplete complex.

Biological applications and some speculations on the evolution of the genetic code will be discussed elsewhere.

Acknowledgements. The author thanks Prof. M. Kotani and Dr. F. Egami for their kind advice. He also acknowledges the linguistic help from Prof. J. Meek. 


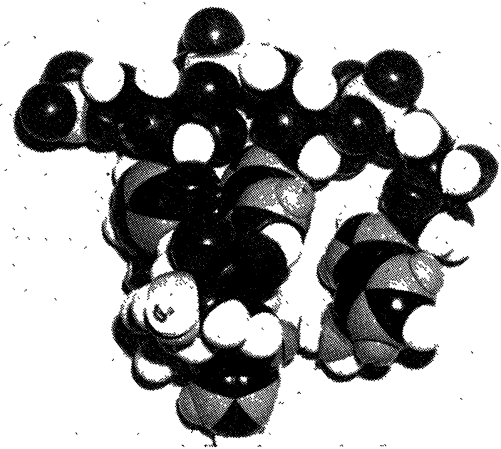

Fig. 5. $\mathrm{C} 4 \mathrm{~N}$ of $\mathrm{UAA} \equiv \mathrm{A}$ with isoleucine on it.

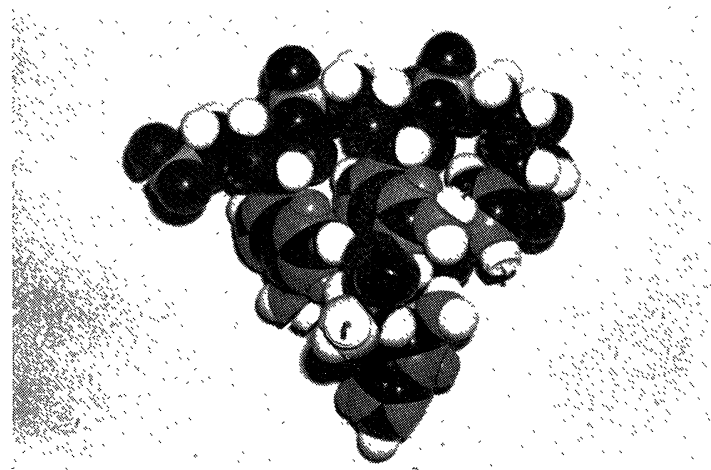

Fig. 6. $\mathrm{C} 4 \mathrm{~N}$ of $\mathrm{AGU} \equiv \mathrm{G}$ with serine $_{4}$ on it.

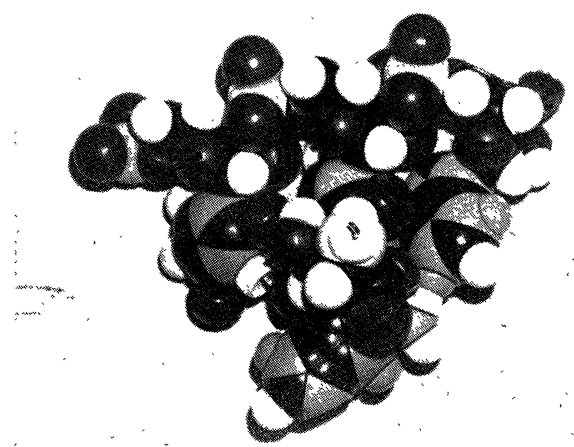

Fig. 7. $\mathrm{C} 4 \mathrm{~N}$ of $\mathrm{UCA} \equiv \mathrm{G}$ with serine $e_{2}$ on it. 


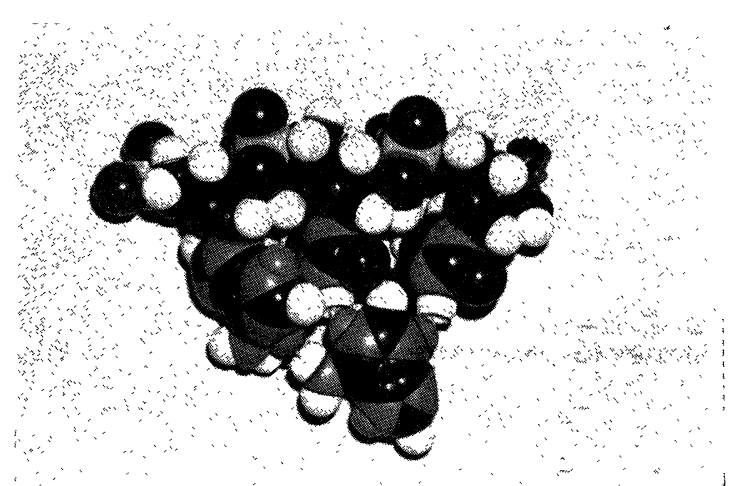

Fig. 8. $\mathrm{C} 4 \mathrm{~N}$ of $\mathrm{AUU} \equiv \mathrm{A}$ for a chain terminator. The hole is too small for all the side chains of protein amino acids.

\section{References}

1) Crothers, D. M., Seno, T., and Soll, D. G. (1972) : Proc. Natl. Acad. Sci. USA, 69, 3063.

2) Wetzel, R. (1978): Origin of Life, $\mathbf{9}, 39$.

3) Hopfield, J. J. (1978) : Proc. Natl. Acad. Sci. USA, 75, 4374.

4) Hayashi, H., and Miura, K. (1966): Nature, 278, 137. 